\title{
DIAGNÓSTICO PARTICIPATIVO PARA DETERMINAR PROBLEMAS AMBIENTALES EN COMUNIDADES RURALES
}

\section{Community participation for the sustainable use of natural resources}

\author{
Adán Guillermo Ramírez-García*, Mirna Camacho-Bercherlt**
}

\section{RESUMEN}

El objetivo del presente trabajo fue identificar los principales problemas ambientales en relación al aprovechamiento de los recursos naturales y los conflictos ambientales que se viven en el municipio de Álamos. Teóricamente se sustenta en los planteamientos de Carabias (1994), Bocco (2000), Chambers, (1983) y Lombera, (2004). Metodológicamente la investigación se soporta en la evaluación rural participativa. De los resultados sobresale la realización de diez talleres, un taller llamado institucional, el cual se realizó con funcionarios y representantes de instituciones de investigación, educación superior, así como de organizaciones no gubernamentales; se llevaron a cabo seis talleres comunitarios que se realizaron con la participación de la población en general de cada comunidad donde se realizó el taller; dos talleres más, uno con ganaderos y otro con silvicultores y finalmente un taller de validación de los resultados. En total se tuvo la participación de 396 personas, 209 mujeres, 187 hombres. Asimismo, se elaboró un mapa donde se ubican los proyectos estratégicos propuestos y se identifican los conflictos que se pueden generar. Se concluye, que de los principales problemas ambientales destacan la escasez y contaminación del agua, la sobreexplotación de los recursos naturales, conflictos en la delimitación de linderos y el cultivo de enervantes.

Palabras clave: Álamos, Ordenamiento, Territorio, Sustentable, Gestión

RECIBIDO: Enero 2018 ACEPTADO: Noviembre 2018

\footnotetext{
* Profesor Investigador de la Universidad Autónoma Chapingo en el Centro Regional Universitario del Noroeste. Ciudad Obregón, Sonora, México. Correo electrónico: gramirezg@taurus.chapingo.mx

** Consultora. Maestría en Ciencias en Estrategia Agroempresarial, Universidad Autónoma Chapingo. Texcoco, estado de México, México. Correo electrónico: agroempresarial.vidasc@gmail.com
} 


\section{ABSTRACT}

The objective of this work was to identify the main environmental problems in relation to the use of natural resources and environmental conflicts that are experienced in the municipality of Álamos. Theoretically it is based on the approaches of Carabias (1994), Bocco (2000), Chambers, (1983) and Lombera, (2004). Methodologically, research is supported in participatory rural appraisal. Out of the results, ten workshops were held, a workshop called institutional, which was carried out with officials and representatives of research institutions, higher education, as well as non-governmental organizations; Six community workshops were held with the participation of the general population of each community where the workshop was held; two more workshops, one with farmers and another with foresters and finally a workshop to validate the results. In total there was the participation of 396 people, 209 women, 187 men. Likewise, a map was drawn up where the proposed strategic projects are located and the conflicts that can be generated are identified. It is concluded that the main environmental problems include the scarcity and contamination of water, overexploitation of natural resources, conflicts in the delimitation of boundaries and the cultivation of drugs.

Key Words: Álamos, Ordering, Territory, Sustainable, Management

\section{Introducción}

En México, generalmente las comunidades rurales, debido a las condiciones de pobreza en que se encuentran, se ven obligados a realizar una explotación intensiva de sus recursos y a utilizar en determinadas actividades predios que no tienen el potencial adecuado (Carabias et al., 1994). Las comunidades rurales presentan fuerte dependencia de los recursos naturales presentes en el entorno en su medio de vida, haciéndolas más propensas a buscar estrategias de vida que garanticen la continuidad de sus recursos a largo plazo, cuando tales recursos están bajo su control (Herman et al., 2003).

Por ello, se considera que las comunidades rurales dependen en gran medida de sus recursos naturales, lo que demuestra su importancia para resguardarlos, ellas pueden operar como aliadas de la protección biológica y la diversidad genética in situ (Bocco et al., 2000). La meta principal de la política ambiental es la construcción de 
Diagnóstico participativo para determinar problemas ambientales en comunidades rurales

una sociedad donde el desarrollo sustentable sea posible. La planeación del desarrollo local con participación social, es decir la planeación participativa, coadyuva al desarrollo de un ejercicio democrático de gobierno que garantiza procesos de participación ciudadana en la gestión corresponsable de estrategias de desarrollo socio-económico y de políticas públicas locales y, por ende, en la concreción de mejores condiciones integrales de vida para la población (Lombera, 2004).

Estrella y Gaventa (2001) plantean que en los últimos años el término "participación" ha sido un concepto clave en el campo del desarrollo. A nivel internacional los donantes (gobiernos y ONG's) promueven el uso de métodos participativos para evaluar necesidades e implementar programas; asimismo Chambers (1997), menciona que este nuevo enfoque parte del conocimiento de la gente como base para la planeación y el cambio.

La participación social y el empoderamiento o apropiación de las sociedades regionales en las acciones realizadas, son elementos importantes para conseguir efectos sustantivos en el mejoramiento de las zonas marginadas. Un aspecto que debe de cuidarse por su relevancia en el proceso, es que la participación debe ser incluyente, plural y democrática, para evitar el clientelismo, producto de la articulación parcial y políticamente sesgada entre instituciones y organizaciones de la sociedad (Toledo y Bartra, 2000).

En las propuestas de desarrollo alternativo, la participación es un ingrediente fundamental de apropiación y empoderamiento, para que sean las propias comunidades las que dirijan su propio destino de desarrollo. Una activa participación social y el real involucramiento de las comunidades locales son premisas imprescindibles, para las propuestas que armonizan los elementos económicos, sociales y ambientales (García y Rodríguez, 2006).

La información es recogida, sistematizada y analizada, por la propia comunidad, siendo el papel del investigador de mero facilitador. 
Adán Guillermo Ramírez-García y Mirna Camacho-Bercherlt

Telos Vol. 21, No. 1 (2019). 86-113

La acción participativa implica, primero, transmitir la información obtenida al resto de la comunidad u otras organizaciones, mediante reuniones, representaciones teatrales u otras técnicas, y, además, con frecuencia, llevar a cabo acciones para transformar la realidad. d) La evaluación, sea mediante los sistemas ortodoxos en las ciencias sociales o simplemente estimando la efectividad de la acción en cuanto a los cambios logrados, por ejemplo, en cuanto al desarrollo de nuevas actitudes, o la redefinición de los valores y objetivos del grupo (Guzmán et. al, 1994).

La gestión de la comunidad en los recursos naturales se ha promovido extensivamente en años recientes como un acercamiento para perseguir la conservación biológica y el logro objetivos socio-económicos (Stephen et al., 2000).

La evaluación rural participativa (ERP) estima que la colaboración social es un ingrediente fundamental para elaborar proyectos, impulsar un mejoramiento sustantivo en la calidad de vida local y conservar los recursos naturales. El método de ERP derivó de la evaluación rural rápida (ERR) y se utilizó por primera vez a comienzos de la década de los ochenta (Chambers, 1983). La ERP sólo podrá ser útil cuando se den estas condiciones fundamentales para la negociación, donde las comunidades están convencidas de participar.

La práctica de la investigación e innovación participativas, entre los pequeños productores agropecuarios, es un recurso interesante ya que aprovecha el conocimiento empírico que tienen los productores, no sólo de los recursos naturales con que cuentan y su manejo, sino también de las potencialidades y los límites que impone el ambiente cultural, social y político en que sus explotaciones están insertas (García y Rodríguez, 2006).

Investigaciones llevadas a cabo sobre la aplicación del enfoque participativo en la transferencia tecnológica y la innovación con pequeños productores para problemas productivos concretos, han mostrado que los campesinos adquieren mayor confianza y se involucran más rápido que en el modelo convencional, fomentando 
Diagnóstico participativo para determinar problemas ambientales en comunidades rurales

además la formación de redes de comunicación entre campesinos y científicos para resolver problemas (Córdoba, et al. 2004).

La investigación participativa, puede contribuir al fortalecimiento de las capacidades locales, generar más confianza en los participantes y a incrementar conocimientos. Su papel consiste en la producción de información y a la experimentación con diferentes métodos y enfoques que contribuyan a la generación de herramientas e iniciativas para el empoderamiento (Hellin y Badstue, 2006).

Existen diferentes técnicas y metodologías que implementan el enfoque de investigación participativa, las cuales son: El Diagnóstico Rural Participativo, La metodología “Sondeo”, Diagnóstico Rural Rápido, Entrevistas, Planeación Estratégica Participativa, Talleres Participativos (Contreras et al., 1998; FAO 1996; Lombera, 2004).

La promoción de la participación comunitaria en las actividades de conservación y uso racional de los recursos representa otra estrategia que puede ayudar a resolver la problemática presentada en un territorio o comunidad. Si no existe concertación con los habitantes y usuarios de un territorio las actividades de manejo y operación del área no pueden llevarse a cabo (Scheinfeld, 1999).

Los procesos de la participación comprenden un conjunto de acciones que tienen como objetivo, informar, difundir y sensibilizar acerca del estado actual de la localidad, brindando argumentos para lograr el desarrollo sustentable de la región, con el fin de involucrar a todos los actores en la participación del proceso de planificación (Mormontoy et al. 2006). La participación no es un estado fijo, es un proceso mediante el cual la gente puede ganar más o menos grados de participación en el proceso de desarrollo (Geilfus, 1997).

Miranda y Matos (2002), sostienen que el planeamiento para el desarrollo sostenible a nivel local es un proceso participativo de carácter técnico y político: "La naturaleza política deviene del hecho de que toda decisión y definición de objetivos pasa por múltiples y diversificados intereses $\mathrm{y}$, por tanto, envuelve conflictos $\mathrm{y}$ 
Adán Guillermo Ramírez-García y Mirna Camacho-Bercherlt

Telos Vol. 21, No. 1 (2019). 86-113

requiere negociaciones entre actores sociales. Se trata de un proceso dialéctico inherente al método participativo, ascendente y descentralizado de planeamiento.

Los conflictos sobre los recursos naturales son desacuerdos y contiendas sobre el acceso, control y utilización de éstos. Los conflictos surgen a menudo porque la gente utiliza los recursos naturales (bosques, agua, pastos y tierra), cada quien, de distinta manera, o porque deciden cambiar la forma de manejarlos. Los desacuerdos también surgen cuando estos intereses y necesidades son incompatibles, o cuando las prioridades de algún grupo de usuarios no figuran dentro de las políticas, programas y proyectos. Tales conflictos de interés son una característica inevitable de todas las sociedades (FAO, 2001).

Selman (2004), afirma que la participación comunitaria resulta indispensable en cualquier proceso de planeación de recursos naturales, difícilmente las iniciativas tomadas por la comunidad pueden sustituir a la planeación formal de extensas áreas. Sin embargo, la conjunción de ambas resulta útil a la hora de los consensos en la solución de los conflictos que pueden surgir por diferentes intereses. Sin duda, la planeación participativa es una herramienta útil en el diagnóstico de los problemas relacionados al aprovechamiento de los recursos naturales y los conflictos ambientales que se viven en las comunidades rurales. En este contexto, el objetivo del presente trabajo fue identificar los principales problemas ambientales en relación al aprovechamiento de los recursos naturales y los conflictos ambientales que se viven en el municipio de Álamos.

\section{Materiales y Métodos}

\section{1 Área de estudio}

El estudio se realizó en el municipio de Álamos, ubicado al sureste del estado de Sonora, entre los paralelos $27^{\circ} 46.2^{\prime}$ y $26^{\circ} 23.4^{\prime}$ ' de latitud norte y entre los $108^{\circ} 25.2^{\prime}$ y $109^{\circ} 15.6$ ' de longitud oeste, tiene una extensión en superficie de $6,947.47$ km$^{2}$ (Figura 1). Respecto a la Orografía, el $70 \%$ de la superficie total del municipio es zona 
Diagnóstico participativo para determinar problemas ambientales en comunidades rurales

accidentada, localizándose en la región noreste y zonas aisladas donde el terreno está surcado por las derivaciones de la Sierra Madre Occidental. La altura sobre el nivel del mar en esta área varía de 500 a 2,000 metros.

Figura 1. Ubicación geográfica de la zona de estudio.

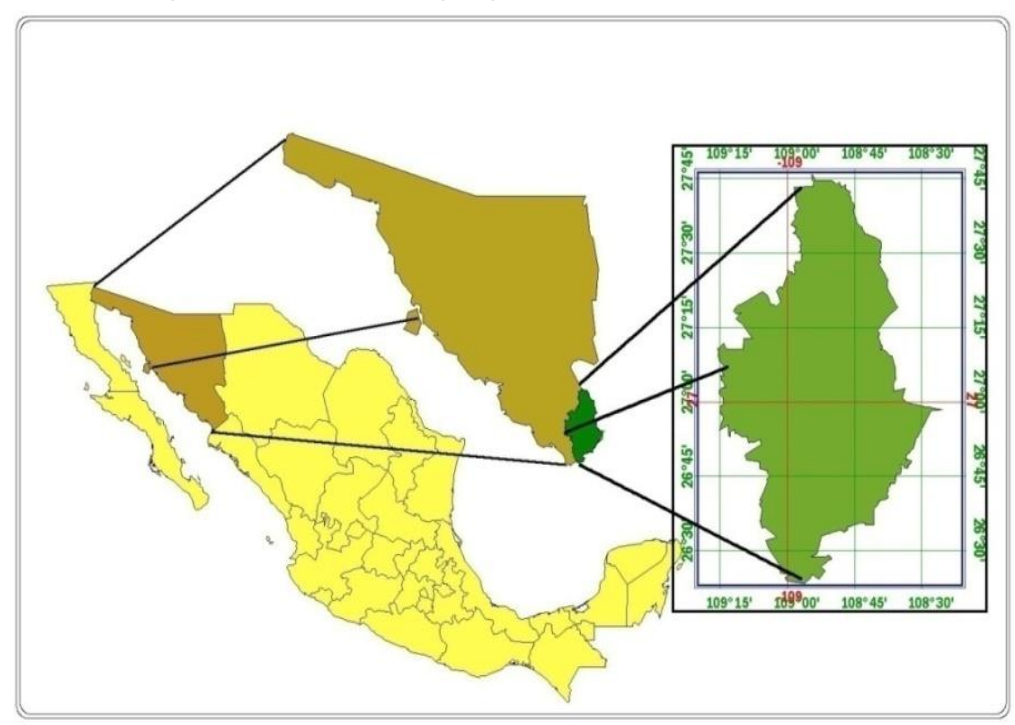

Fuente. Elaboración propia.

De acuerdo con INEGI (2011), la población total del municipio es de 25,848 habitantes, de estos 13,497 son hombres y 12,351 son mujeres. En lo que se refiere a los principales indicadores demográficos del municipio a continuación se desglosan, Cuadro 1.

\section{Cuadro 1. Indicadores demográficos.}

\begin{tabular}{|l|c|}
\hline Número de localidades & 321 \\
\hline (\%) Tasa de crecimiento de la población 2005-2010 & -0.99 \\
\hline \% de la población urbana & 36.15 \\
\hline \% de la población rural & 63.84 \\
\hline Edad mediana de la población (años) & 28 \\
\hline Densidad de población (hab/km $\left.{ }^{2}\right)$ & 3.7 \\
\hline
\end{tabular}


Adán Guillermo Ramírez-García y Mirna Camacho-Bercherlt

Telos Vol. 21, No. 1 (2019). 86-113

Fuente: Elaboración propia con base en COESPO, 2010. Indicadores Demográficos y

Socioeconómicos del estado de Sonora.

En cuanto a la distribución de la población por tamaño de la localidad se presenta en la Cuadro 2.

Cuadro 2. Número de localidades por tamaño y población.

\begin{tabular}{|c|c|c|c|c|c|c|c|c|c|c|}
\hline \multirow{2}{*}{\multicolumn{2}{|c|}{ Municipio }} & \multicolumn{9}{|c|}{ Habitantes por localidad } \\
\hline & & \multirow{2}{*}{$\begin{array}{r}1 \text { a } 49 \\
234\end{array}$} & \multirow{2}{*}{$\begin{array}{c}50 \text { a } 99 \\
29\end{array}$} & \multirow{2}{*}{$\begin{array}{c}100 \\
a \\
499 \\
\\
\\
\\
50\end{array}$} & \multirow{2}{*}{$\begin{array}{c}500 \\
a \\
999 \\
\\
\\
\end{array}$} & \multirow{2}{*}{$\begin{array}{c}1000 \\
a \\
1999 \\
\\
\\
1\end{array}$} & \multirow{2}{*}{$\begin{array}{c}2000 \\
a \\
2499 \\
\\
\\
0\end{array}$} & \multirow{2}{*}{$\begin{array}{c}2500 \\
a \\
4999 \\
\\
\\
\\
0\end{array}$} & \multirow{2}{*}{$\begin{array}{c}5000 \\
a \\
9999 \\
\\
\\
1\end{array}$} & \multirow{2}{*}{$\begin{array}{c}\text { Más de } \\
1000\end{array}$} \\
\hline Localidades & 317 & & & & & & & & & \\
\hline Población & 25,848 & 2,018 & 2,094 & 10,071 & 1,253 & 1,067 & 0 & 0 & 9,345 & 0 \\
\hline
\end{tabular}

Fuente: Elaboración propia con base en INEGI (2011). II Conteo de Población y Vivienda.

Bajo estas consideraciones se ubicaron las comunidades dónde se trabajó, dado que las distancias entre ellas y la densidad de población fue un factor que limito se pudieran abordar más localidades.

\section{Metodología}

Para la evaluación rural participativa, se consideraron principalmente tres estrategias metodológicas.

1. Recorridos de campo: a través de los denominados transectos se hicieron recorridos de campo con un grupo de entre tres y cuatro habitantes de las comunidades donde se realizaron los talleres, en total fueron 6 recorridos de campo. Se buscaba que tuvieran conocimientos generales sobre flora y fauna, así como de lugares emblemáticos de la comunidad y sobre la problemática relacionada con los recursos naturales y/o que podían hacer algún aporte de interés al proyecto. Las rutas se definieron en base a los mapas generados en los talleres participativos. 
Diagnóstico participativo para determinar problemas ambientales en comunidades rurales

2. Entrevistas semiestructuradas: Con información de los talleres participativos y los recorridos de campo fue necesario ubicar informantes clave, autoridades de la comunidad, responsables del ayuntamiento, investigadores del tema, entre otros, para precisar aspectos puntuales de la problemática ambiental, con el fin triangular información desde diferentes puntos de vista y que permitieran precisar los problemas, conflictos y en su caso, las posibles alternativas. Se consideró líderes de las comunidades, investigadores del tema, así como algunos prestadores de servicios profesionales que trabajaban en algún programa de Gobierno.

En cuanto a la los resultados de los recorridos de campo y las entrevistas semiestructuradas, se plasman en la identificación de conflictos ambientales, conclusiones y alternativas. Resaltando que fue un medio para verificar lo que se encontró en los talleres participativos. A continuación, se señala la cantidad de personas en cada caso y su clasificación, Cuadro 3.

Cuadro 3. Participantes en las entrevistas estructuradas.

\begin{tabular}{|l|c|}
\hline \multicolumn{1}{|c|}{ Clasificación } & \multicolumn{1}{|c|}{$\begin{array}{c}\text { Cantidad de } \\
\text { participantes }\end{array}$} \\
\hline Comisariado ejidal (uno por comunidad) & 6 \\
\hline $\begin{array}{l}\text { Representante de instituciones (Sedesol 2, Semarnat 1, Conanp 5, Conafor 3, } \\
\text { Ayuntamiento 4) }\end{array}$ & 15 \\
\hline Académicos (Unison 4, Itson 4, Cruno 2) & 10 \\
\hline Prestador de servicios profesionales (uno por comunidad) & 6 \\
\hline $\begin{array}{l}\text { Representantes de organizaciones (representantes de prestadores de servicios } \\
\text { turísticos 3. }\end{array}$ & 3 \\
\hline \multicolumn{2}{|c|}{ Total }
\end{tabular}

Fuente: Elaboración propia.

3. Talleres participativos: el objetivo general de los talleres fue propiciar la participación de los diferentes actores mediante la recolección y análisis conjunto de la información sobre aspectos, sociales, económicos, ambientales e institucionales. En cada una de las comunidades donde se realizaron estos talleres, se hacia la invitación abierta a toda la comunidad incluidos hombre y 
mujeres de todas las edades. Para el caso de los talleres con instituciones y por sector, la invitación era dirigida. En los talleres institucionales eran principalmente funcionarios de dependencias como Secretaría de Agricultura, de Medio Ambiente, de Áreas Naturales, del Ayuntamiento y de la Secretaría de Desarrollo Social. Mientras que en los talleres por sector los asistentes principalmente eran miembros de organizaciones dedicadas a las actividades propias del taller.

El proceso de evaluación rural participativa tuvo los siguientes objetivos:

1. Identificar mediante la información obtenida en los talleres participativos, aspectos relevantes sobre producción, población, tenencia y uso de la tierra, que den elementos que permitan la planeación del uso sustentable de los recursos naturales.

2. Identificar aspectos generadores de conflicto, formas de expresión y sistemas de resolución de los mismos a nivel regional.

3. Establecer de acuerdo con los puntos anteriores, los criterios básicos para la complementar la propuesta de planeación del territorio.

4. Contribuir a la elaboración del plan de acción y las estrategias de trabajo para implementar el modelo de desarrollo.

\section{Resultados y Discusión}

\section{Diagnóstico participativo}

El desarrollo rural tiene que estudiarse como un estado dinámico en el que la participación hace posible pasar gradualmente de una pasividad casi completa al control de su propio proceso, (Geilfus, 1997). La utilización frecuente de talleres participativos en actividades de diagnósticos para la gestión del desarrollo rural, se debe principalmente a que se reconoce que estos procesos no son lineales y al contrario son complejos. Son considerados aquellos espacios para llevar a cabo la 
Diagnóstico participativo para determinar problemas ambientales en comunidades rurales

discusión y reflexión con los diferentes actores sociales. Se emplearon como insumos el conocimiento y la experiencia de los asistentes, las herramientas fueron las técnicas de dinámica de grupos, visualización, entrevistas y comunicación oral y de observación de campo (Ramírez, et al, 2012).

El taller fue un escenario creado para producir, analizar e intercambiar, en tiempos definidos puntos de vista sobre:

- Experiencias e información acerca de las necesidades locales, los problemas y la situación que guardan los recursos naturales del municipio.

- El proceso de transformación ambiental que se manifiesta en la región.

- Las alternativas que instituciones, comunidad y técnicos pueden encontrar para ayudar a satisfacer algunas necesidades sentidas, $\mathrm{y}$

- Como mejorar o restaurar en términos generales, esa situación ambiental y contribuir a superar el propio desarrollo productivo.

Los talleres que se llevaron a cabo fueron con instituciones municipales, ejidatarios forestales, así como ganaderos privados y ejidales. En cada taller se registró una relatoría donde se anotaron los pormenores de cada día de actividad. Se realizaron un total de 10 talleres; un taller llamado institucional, el cual se realizó con funcionarios y representantes de instituciones de investigación, educación superior, así como de organizaciones no gubernamentales; seis talleres comunitarios que se realizaron con la participación de la población en general de cada comunidad; dos talleres más, uno con ganaderos y otro con silvicultores y finalmente, un taller de validación de los resultados donde participaron asistentes de todos los talleres mencionados, en total se tuvo la participación de 396 personas, 209 mujeres, 187 hombres, Cuadro 4. 
Adán Guillermo Ramírez-García y Mirna Camacho-Bercherlt

Telos Vol. 21, No. 1 (2019). 86-113

Cuadro 4. Talleres participativos realizados.

\begin{tabular}{|c|c|c|c|c|c|}
\hline Tipo de taller & $\begin{array}{c}\text { Talleres } \\
\text { realizados }\end{array}$ & Asistentes & $\begin{array}{l}\text { Lugar de } \\
\text { realización }\end{array}$ & $\begin{array}{c}\text { Días de } \\
\text { Duración }\end{array}$ & $\begin{array}{c}\text { Cantidad } \\
\text { de } \\
\text { asistentes }\end{array}$ \\
\hline Institucional & 1 & $\begin{array}{l}\text { Representantes } \\
\text { de instituciones, } \\
\text { organizaciones } \\
\text { de la sociedad } \\
\text { civil, } \\
\text { autoridades } \\
\text { municipales }\end{array}$ & Álamos & 2 & 32 \\
\hline \multirow{6}{*}{ Comunitarios } & \multirow{6}{*}{6} & $\begin{array}{l}\text { Ejidatarios, } \\
\text { colonos y } \\
\text { avecindados, } \\
\text { comisariado } \\
\text { ejidal }\end{array}$ & $\begin{array}{l}\text { Piedras } \\
\text { Verdes }\end{array}$ & 2 & 43 \\
\hline & & $\begin{array}{l}\text { Ejidatarios, } \\
\text { colonos } \\
\text { avecindados, } \\
\text { comisariado } \\
\text { ejidal }\end{array}$ & $\begin{array}{c}\text { La } \\
\text { Aduana }\end{array}$ & 2 & 35 \\
\hline & & $\begin{array}{l}\text { Ejidatarios, } \\
\text { colonos y } \\
\text { avecindados, } \\
\text { comisariado } \\
\text { ejidal }\end{array}$ & La Labor & 2 & 33 \\
\hline & & $\begin{array}{l}\text { Ejidatarios, } \\
\text { colonos } \\
\text { avecindados, } \\
\text { comisariado } \\
\text { ejidal }\end{array}$ & $\begin{array}{l}\text { Potrero } \\
\text { de } \\
\text { Alcantar }\end{array}$ & 2 & 39 \\
\hline & & $\begin{array}{l}\text { Ejidatarios, } \\
\text { colonos } \\
\text { avecindados, } \\
\text { comisariado } \\
\text { ejidal }\end{array}$ & $\begin{array}{c}\text { El Cajón } \\
\text { del } \\
\text { Sabino }\end{array}$ & 2 & 38 \\
\hline & & $\begin{array}{l}\text { Ejidatarios, } \\
\text { colonos } \\
\text { avecindados, } \\
\text { comisariado } \\
\text { ejidal }\end{array}$ & El Zapote & 2 & 36 \\
\hline \multirow{2}{*}{ Por sector } & \multirow{2}{*}{2} & Ganaderos & Álamos & 2 & 39 \\
\hline & & Silvicultores & Álamos & 2 & 34 \\
\hline Validación & 1 & $\begin{array}{l}\text { Comisarios } \\
\text { ejidales, } \\
\text { representantes }\end{array}$ & Álamos & 2 & 67 \\
\hline
\end{tabular}

Fuente: Elaboración propia. 
Diagnóstico participativo para determinar problemas ambientales en comunidades rurales

El objetivo general de los talleres fue propiciar la participación de los diferentes actores mediante la recolección y análisis conjunto de la información sobre aspectos sociales, económicos, ambientales e institucionales. Los objetivos particulares de los talleres donde participó el personal de las instituciones consistieron en abordar diferentes temas para esbozar proyectos estratégicos que contribuyan al desarrollo sustentable del municipio; además de analizar su importancia para evaluar y programar el uso del suelo y el manejo de los recursos naturales; asimismo, determinar los recursos naturales con los que se cuenta y analizar los problemas ambientales, sociales y económicos que tiene el municipio. Los actores locales, papel en el proceso participativo y asistente al taller se pueden observar en el Cuadro 5.

Cuadro 5. Actores participantes en los talleres del proceso participativo.

\begin{tabular}{|c|c|c|c|}
\hline Actor & $\begin{array}{l}\text { Papel en el } \\
\text { proceso } \\
\text { Participativo }\end{array}$ & $\begin{array}{l}\text { Asistente al } \\
\text { taller }\end{array}$ & $\begin{array}{l}\text { Cantidad de } \\
\text { asistentes }\end{array}$ \\
\hline Instituciones & $\begin{array}{l}\text { Tienen la potestad } \\
\text { de } \\
\text { decisiones tomar } \\
\text { cuentan con } \\
\text { diversos programas } \\
\text { de apoyo y } \\
\text { subsidio. Operan la } \\
\text { política pública a a } \\
\text { nivel nacional, } \\
\text { estatal y municipal. }\end{array}$ & $\begin{array}{l}\text { SEDESOL, } \\
\text { CONANP- } \\
\text { SEMARNAP, } \\
\text { APFF "Sierra de } \\
\text { Álamos, } \\
\text { Ayuntamiento, } \\
\text { CNA, Municipio de } \\
\text { Álamos, } \\
\text { SAGARPA, } \\
\text { Regidor Étnico } \\
\text { Guarijío. }\end{array}$ & 32 \\
\hline Comunidad & $\begin{array}{l}\text { Aportan elementos } \\
\text { importantes en } \\
\text { base a su } \\
\text { experiencia } \\
\text { conocimiento } \\
\text { empírico. Además } \\
\text { que son los dueños } \\
\text { de la tierra. }\end{array}$ & $\begin{array}{lr}\text { S.S.S. } & \text { U.M.A., } \\
\text { APFF, } & \text { P.S.P. } \\
\text { ciudadanos } & \text { de } \\
\text { diferentes } & \\
\text { comunidades. }\end{array}$ & 275 \\
\hline Académicos & $\begin{array}{l}\text { Toma de } \\
\text { decisiones en la } \\
\text { creación } \\
\text { escenarios de } \\
\text { propuestas, en la } \\
\text { evaluación técnica, } \\
\text { social y económica } \\
\text { de las acciones } \\
\text { propuestas. }\end{array}$ & $\begin{array}{l}\text { ITSON Navojoa y } \\
\text { Obregón, } \\
\text { COBACH Álamos, } \\
\text { Investigadora } \\
\text { proyecto sierra } \\
\text { mar AC., CRUNO- } \\
\text { UACH. }\end{array}$ & 16 \\
\hline
\end{tabular}


Adán Guillermo Ramírez-García y Mirna Camacho-Bercherlt

Telos Vol. 21, No. 1 (2019). 86-113

\begin{tabular}{|c|c|c|c|}
\hline Por sector & $\begin{array}{l}\text { Los gremios } \\
\text { económicos } \\
\text { contribuyen a la } \\
\text { proyección regional } \\
\text { del territorio. } \\
\text { Generan empleo. }\end{array}$ & $\begin{array}{l}\text { Anfitriones } \\
\text { turísticos, } \\
\text { asociación } \\
\text { ganadera local y } \\
\text { forestal, ejidatarios } \\
\text { de diferentes } \\
\text { comunidades }\end{array}$ & 73 \\
\hline
\end{tabular}

Fuente: Elaboración propia.

Trabajo en equipo en los talleres participativos: El trabajo en equipo tuvo como fin compartir la información generada por los asistentes. Los equipos se formaron generando diversidad en su conformación y procurando que no existieran grupos de integrantes de una misma institución, ejido, comunidad o género. Se trabajó en plenaria la presentación de resultados, en la discusión de un tema en particular o alguna presentación del equipo facilitador. Las decisiones se tomaron en consenso.

Las herramientas se seleccionaron y diseñaron fundamentalmente en función de los objetivos del taller, de las características de los actores sociales con los que se trabajó en cada uno de los talleres, al tema definido, al lugar de aplicación del taller y de las condiciones climáticas de la temporada; en el Cuadro 6, se presentan temas, objetivos y dinámicas de ERP utilizadas en los diferentes talleres.

Cuadro 6. Temas, Objetivos y dinámicas utilizadas en los talleres.

\begin{tabular}{|l|l|l|}
\hline \multicolumn{1}{|c|}{ Tema } & \multicolumn{1}{c|}{ Objetivo } & \multicolumn{1}{c|}{ Dinámicas } \\
\hline Inicio de la sesión & $\begin{array}{l}\text { "Romper el hielo" para iniciar la } \\
\text { sesión }\end{array}$ & La telaraña \\
\hline $\begin{array}{l}\text { Expectativas del curso } \\
\text { taller }\end{array}$ & $\begin{array}{l}\text { Conocer los intereses y } \\
\text { expectativas de los asistentes }\end{array}$ & $\begin{array}{l}\text { Pregunta: ¿Qué } \\
\text { espero del taller? }\end{array}$ \\
\hline Objetivos & $\begin{array}{l}\text { Informar sobre los objetivos del } \\
\text { curso taller y precisar sus } \\
\text { alcances }\end{array}$ & Plenaria \\
\hline $\begin{array}{l}\text { Importancia de la } \\
\text { planeación para el } \\
\text { aprovechamiento de los } \\
\text { recursos naturales }\end{array}$ & $\begin{array}{l}\text { Mostrar la importancia de la } \\
\text { planeación y analizar en qué } \\
\text { consiste. }\end{array}$ & Exposición \\
\hline $\begin{array}{l}\text { Diagnóstico } \\
\text { comunitario }\end{array}$ & $\begin{array}{l}\text { Practicar una herramienta } \\
\text { participativa para hacer un } \\
\text { diagnóstico comunitario }\end{array}$ & Mapa Base \\
\hline Diagnóstico Social y de & Realizar un diagnóstico de los & Diagrama de Venn \\
\hline
\end{tabular}


Diagnóstico participativo para determinar problemas ambientales en comunidades rurales

\begin{tabular}{|c|c|c|}
\hline organizaciones & $\begin{array}{l}\text { actores sociales y complementar } \\
\text { las dos actividades anteriores }\end{array}$ & \\
\hline $\begin{array}{l}\text { Actividades y } \\
\text { proyectos que realiza } \\
\text { cada institución }\end{array}$ & $\begin{array}{l}\text { Conocer cómo participan dentro } \\
\text { del desarrollo municipal }\end{array}$ & $\begin{array}{l}\text { Diagrama de } \\
\text { actividades y } \\
\text { proyectos por } \\
\text { institución }\end{array}$ \\
\hline $\begin{array}{l}\text { Calendario estacional } \\
\text { de actividades } \\
\text { productivas }\end{array}$ & $\begin{array}{l}\text { Representar el calendario de } \\
\text { actividades productivas }\end{array}$ & $\begin{array}{l}\text { Calendario } \\
\text { estacional }\end{array}$ \\
\hline Especies aprovechadas & $\begin{array}{l}\text { Identificar las especies } \\
\text { forestales aprovechadas y su } \\
\text { potencial }\end{array}$ & $\begin{array}{l}\text { Uso local de } \\
\text { recursos naturales }\end{array}$ \\
\hline $\begin{array}{l}\text { Problemas de la } \\
\text { comunidad y de la } \\
\text { actividad ganadera }\end{array}$ & $\begin{array}{l}\text { Conocer las causas y las } \\
\text { consecuencias de los problemas } \\
\text { expuestos }\end{array}$ & Árbol de problemas \\
\hline $\begin{array}{l}\text { Problemas de la } \\
\text { actividad forestal }\end{array}$ & $\begin{array}{l}\text { Conocer los problemas más } \\
\text { relevantes de la actividad } \\
\text { forestal }\end{array}$ & Árbol de problemas \\
\hline $\begin{array}{l}\text { Priorización de } \\
\text { problemas }\end{array}$ & $\begin{array}{l}\text { Exponer algunas ideas sobre la } \\
\text { importancia del trabajo en } \\
\text { equipo }\end{array}$ & $\begin{array}{l}\text { Matriz de } \\
\text { priorización }\end{array}$ \\
\hline $\begin{array}{l}\text { Conflicto en el manejo } \\
\text { de recursos naturales }\end{array}$ & $\begin{array}{l}\text { Identificar cuáles y en dónde se } \\
\text { presentan conflictos producto } \\
\text { del manejo y aprovechamiento } \\
\text { de recursos naturales }\end{array}$ & $\begin{array}{l}\text { Matriz de análisis } \\
\text { de conflictos }\end{array}$ \\
\hline $\begin{array}{l}\text { Propuesta de proyectos } \\
\text { estratégicos } \\
\text { Municipal con } \\
\text { Instituciones } \\
\text { Forestal } \\
\text { Ganadero } \\
\end{array}$ & $\begin{array}{l}\text { Presentar propuesta } \\
\text { Municipal } \\
\text { Actividad forestal } \\
\text { Actividad ganadera }\end{array}$ & $\begin{array}{l}\text { Mapa de ubicación } \\
\text { de proyectos } \\
\text { estratégicos }\end{array}$ \\
\hline Proyectos y actividades & $\begin{array}{l}\text { Delinear los proyectos y } \\
\text { actividades que se consideran } \\
\text { como importantes y necesarios }\end{array}$ & $\begin{array}{l}\text { Proyectos para el } \\
\text { desarrollo de la } \\
\text { actividad forestal y } \\
\text { ganadera }\end{array}$ \\
\hline $\begin{array}{l}\text { Reflexión final y } \\
\text { evaluación }\end{array}$ & $\begin{array}{l}\text { Intercambio de puntos de vista y } \\
\text { evaluación del taller por los } \\
\text { asistentes }\end{array}$ & Plenaria \\
\hline
\end{tabular}

Fuente: Elaboración propia, en base a los talleres participativos. 
Adán Guillermo Ramírez-García y Mirna Camacho-Bercherlt

Telos Vol. 21, No. 1 (2019). 86-113

Programación de las actividades a realizar en los talleres: Cada uno de los talleres a desarrollar se discutieron al interior del equipo de trabajo los objetivos, temas, herramientas, material a utilizar, así como los resultados esperados; se determinó quién fungiría como facilitador y el tiempo dedicado a cada una de las actividades, todo esto queda detallado en las cartas descriptivas, en una secuencia lógica. En base a los objetivos y a la metodología planteada en los talleres participativos realizados con instituciones, con productores forestales y ganaderos, se obtuvieron los siguientes resultados.

\section{a. Con las instituciones}

El diagnóstico realizado por medio de los talleres participativos permitió a los habitantes visualizar las amenazas y debilidades que tienen, pero también las oportunidades que pueden aprovechar y convertir en fortalezas. Los representantes de las diferentes instituciones que asistieron al taller lograron identificar las principales actividades productivas, problemática y riquezas naturales dentro de los diferentes poblados pertenecientes al municipio de Álamos, Sonora, es decir, realizar un diagnóstico actual de la región, Cuadro 7.

Cuadro 7. Actividades productivas, problemática y riquezas naturales.

\begin{tabular}{|l|l|l|l|}
\hline \multicolumn{1}{|c|}{$\begin{array}{c}\text { Actividad } \\
\text { productiva }\end{array}$} & \multicolumn{1}{|c|}{$\begin{array}{c}\text { Lugar donde se } \\
\text { práctica }\end{array}$} & Problemática & \multicolumn{1}{c|}{$\begin{array}{c}\text { Recurso } \\
\text { natural utilizado }\end{array}$} \\
\hline Ganadería & Todo el municipio & $\begin{array}{l}\text { *Explotación } \\
\text { ganadera extensiva } \\
\text { * Sobrepastoreo }\end{array}$ & $\begin{array}{l}\text { Pastizal } \\
\text { Agua }\end{array}$ \\
\hline Agricultura & $\begin{array}{l}\text { Forma temporal } \\
\text { menor escala. } \\
\text { Forma } \\
\text { complementaria }\end{array}$ & Erosión del suelo & $\begin{array}{l}\text { Menor escala: } \\
\text { sorgo forrajero etc. } \\
\text { Complementaria: } \\
\text { aprovechamiento } \\
\text { de palo colorado y } \\
\text { mezquite, etc. }\end{array}$ \\
\hline $\begin{array}{l}\text { Explotación } \\
\text { del } \\
\text { Bosque }\end{array}$ & $\begin{array}{l}\text { Gochico, Chirivo, } \\
\text { Guicochi, } \\
\text { Vinaterías }\end{array} \quad \begin{array}{l}\text { Incendios forestales, } \\
\text { erosión hídrica, } \\
\text { sobrepastoreo, etc. }\end{array}$ & $\begin{array}{l}\text { Pino encino. } \\
\text { Selva } \\
\text { caducifolia }\end{array}$ \\
\hline
\end{tabular}


Diagnóstico participativo para determinar problemas ambientales en comunidades rurales

\begin{tabular}{|l|l|l|l|}
\hline & Baboyahui & y tala de pino. & \\
\hline Minería & $\begin{array}{l}\text { Piedras Verdes, } \\
\text { Zapote, Baboyahui, } \\
\text { Rodeo, Mexiquillo } \\
\text { y Maquipo. }\end{array}$ & $\begin{array}{l}\text { Cobre, plata, oro y } \\
\text { grafito }\end{array}$ & \\
\hline Pesca & $\begin{array}{l}\text { presa del Mocúzari, } \\
\text { presa del Veranito y } \\
\text { arroyo Cuchujaqui }\end{array}$ & $\begin{array}{l}\text { Basura y } \\
\text { contaminación en el } \\
\text { agua }\end{array}$ & \\
\hline Turismo & $\begin{array}{l}\text { Arroyos visitados } \\
\text { por turistas locales } \\
\text { yextranjeros }\end{array}$ & $\begin{array}{l}\text { Descarga de aguas } \\
\text { negras de la Cd. de } \\
\text { Álamos a aguas } \\
\text { naturales de la da } \\
\text { región. }\end{array}$ & $\begin{array}{l}\text { Arroyos, paisajes } \\
\text { naturales. }\end{array}$ \\
\hline $\begin{array}{l}\text { Otras: } \\
\text { Artesanía: }\end{array}$ & $\begin{array}{l}\text { Meza Colorada y } \\
\text { La Aduana. }\end{array}$ & & \\
\hline
\end{tabular}

Fuente: Elaboración propia, en base a los talleres participativos.

En el taller se abordó el tema socioeconómico el cual va de la mano al desarrollo sustentable y es de gran importancia en el proceso participativo. Los problemas que de una u otra forma marcan y definen el rumbo que toma el municipio de Álamos Sonora, se presentan en el Cuadro 8, los cuales fueron las premisas, al igual que las actividades productivas, para la generación de la propuesta de uso del suelo con una visión a 10 años, realizada por los asistentes.

Cuadro 8. Problemática socioeconómica y propuestas.

\begin{tabular}{|l|lll|}
\hline Problemática socioeconómica & \multicolumn{3}{|c|}{ Propuestas } \\
\hline - Pobreza y marginación & - & Carreteras que comuniquen a a \\
- Actitud & & Sinaloa y Chihuahua. \\
- Desempleo & - & Carretera Álamos - Navojoa \\
- Narcotráfico & & carriles \\
- Falta oferta educativa & - & Establecer corredores turísticos \\
- Migración & - Reforestar áreas criticas \\
- Problemas de salud & - & Permiso, vigilancia y seguimiento \\
- Sequía & & en aprovechamiento de recursos \\
- Pérdida de recursos naturales & & forestales. \\
- Corrupción institucional & - Impulsar sector productivo agrícola \\
- Falta de planeación y & & en áreas cerca de arroyos, presas y \\
\hline
\end{tabular}


Adán Guillermo Ramírez-García y Mirna Camacho-Bercherlt

Telos Vol. 21, No. 1 (2019). 86-113

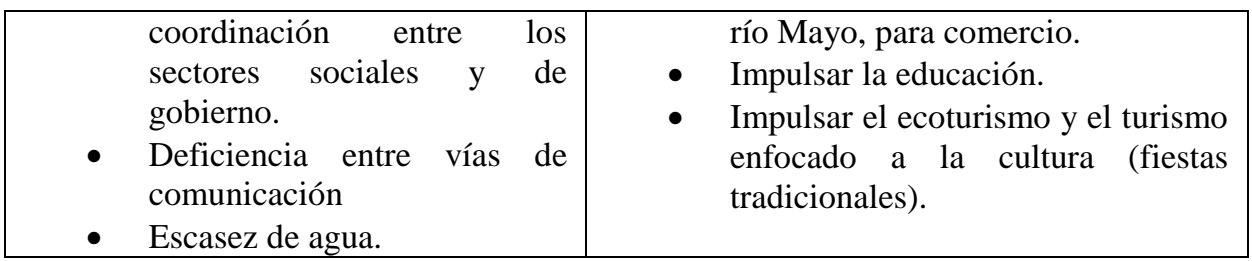

Fuente: Elaboración propia, en base a los talleres participativos.

Los talleres participativos fueron útiles para que los asistentes tomaran conciencia de su situación actual, que no solo es obligación y derecho de las autoridades de los diferentes niveles de gobierno hacer algo por el medio ambiente y por el desarrollo de su región, también les concierne a ellos impulsar dicho proceso.

\section{b. Ganaderos}

En el taller con ganaderos los resultados obtenidos fue producto de su experiencia, saben con precisión los meses en los que se requieren realizar las actividades propias del sector como se muestra en el Cuadro 9, los cuales sirvieron para generar en los ganaderos un estado de reflexión sobre los ciclos del año más productivos, así como las situaciones de conflicto, teniendo como fin propiciar una propuesta de uso del suelo desde su perspectiva.

Cuadro 9. Actividades de la ganadería.

\begin{tabular}{|l|l|}
\hline \multicolumn{1}{|c|}{$\begin{array}{c}\text { Actividades propias de la } \\
\text { ganadería }\end{array}$} & \multicolumn{1}{c|}{ Mes en que se presenta. } \\
\hline Presencia de lluvias & $\begin{array}{l}\text { Junio, julio, agosto y a mediados de } \\
\text { septiembre. Equitapatas: octubre-enero }\end{array}$ \\
\hline Forraje & Mayo, junio y a mediados de julio \\
\hline Pastoreo & $\begin{array}{l}\text { Enero-Abril: sorgo agostadero: agosto a } \\
\text { enero }\end{array}$ \\
\hline Enfermedades & Diciembre, Enero Mayo y Agosto \\
\hline Venta de animales & Noviembre-diciembre ó Enero-febrero \\
\hline Elaboración de panelas & Septiembre-enero \\
\hline $\begin{array}{l}\text { Otras: Vacunas, desparasito y y } \\
\text { Vitaminas para el ganado }\end{array}$ & Marzo-abril y Octubre-noviembre \\
\hline
\end{tabular}


Diagnóstico participativo para determinar problemas ambientales en comunidades rurales

Fuente: Elaboración propia, en base a los talleres participativos.

En el Cuadro 10, se observa la problemática identificada, la solución posible de las actividades propias de la ganadería y la propuesta hechas por los participantes del sector en el taller.

Cuadro 10. Problemática de las actividades propias de la ganadería y propuestas

\begin{tabular}{|c|c|}
\hline $\begin{array}{c}\text { Problemática de las } \\
\text { actividades propias de la } \\
\text { ganadería. }\end{array}$ & Alternativas y propuesta \\
\hline $\begin{array}{ll}\text { - } & \text { Falta de pastura } \\
\text { - } & \text { Falta de agua } \\
\text { - } & \text { pajo poriciones } \\
\text { - } & \text { Falta de capacitación } \\
\text { - } & \text { Perros salvajes } \\
\text { - } & \text { Erosión del suelo } \\
\text { - } & \text { Abigeato } \\
\text { - } & \text { Insumos caros } \\
\text { - } & \text { Comercialización } \\
\text { - } & \text { Manejo de potreros } \\
\text { - Época empadre } \\
\text { - } \quad \text { Lejanía y selección }\end{array}$ & $\begin{array}{l}\text { Soluciones: } \\
>\text { Inversiones en infraestructura y centros } \\
\text { de acopio } \\
>\text { Mejores caminos por medio de programas } \\
>\text { Reforestación } \\
>\text { Construcción de bebederos } \\
>\text { Impulsar el ecoturismo. } \\
\text { Propuesta como organización: } \\
>\text { gestionar ante los organismos } \\
\text { correspondientes y hacer operativos los } \\
\text { programas. } \\
\text { Apoyos que ocupan: } \\
>\text { créditos con interés bajo } \\
>\text { apoyos oficiales de Reforestación } \\
>\text { PET recursos económicos-obras } \\
\text { Su participación como sociedad: } \\
>\text { Organizarse y aprovechar oportunidades } \\
>\text { Establecer áreas de riego } \\
>\text { Diversificar actividad en animales y } \\
>\text { plantas } \\
\text { Ecoturismo: poblados como vinatería. } \\
\text { Posee belleza y variedad de flora y fauna }\end{array}$ \\
\hline
\end{tabular}

Fuente: Elaboración propia, en base a los talleres participativos. 
Adán Guillermo Ramírez-García y Mirna Camacho-Bercherlt

Telos Vol. 21, No. 1 (2019). 86-113

\section{c. Forestal.}

En el Cuadro 11, se muestran los resultados obtenidos en el taller con productores forestales, en donde se logró la identificación de árboles maderables aprovechados en la región, problemática forestal desde su propia perspectiva y se obtuvo una propuesta realizada por ellos mismos, plasmando sus necesidades e intereses.

Cuadro 11. Árboles aprovechados, problemática forestal y alternativas.

\begin{tabular}{|c|c|c|}
\hline Árboles maderables & Problemática forestal & Alternativas \\
\hline $\begin{array}{l}\text { - } \text { Mezquite } \\
\text { - } \text { Palo colorado } \\
\text { - } \text { Amapa } \\
\text { - } \text { Copalquín } \\
\text { - } \text { Palo fierro } \\
\text { - Encino } \\
\text { - } \text { Brasil } \\
\text { - } \text { Mauto } \\
\text { - Otros. }\end{array}$ & $\begin{array}{l}\text { Sequía } \\
\text { Plagas } \\
\text { Erosión } \\
\text { Deforestación } \\
\text { Dobredre explotación } \\
\text { (cultivos enervantes) } \\
\text { * Caminos en mal estado } \\
\text { Falta de: } \\
\text { * Programas de } \\
\text { restauración } \\
\text { * Proyectos alternativos } \\
\text { diversificados } \\
\text { * Asesoría técnica } \\
\text { * Autogestión de } \\
\text { proyectos } \\
\text { * Atención institucional } \\
\text { * Apoyos. }\end{array}$ & $\begin{array}{l}\text { Propuesta como } \\
\text { organización: } \\
>\text { Consolidarse como } \\
\text { organización. } \\
\text { Apoyos que ocupan: } \\
>\text { Asesoría técnica } \\
>\text { identificación de } \\
\text { iniciativas de } \\
\text { desarrollo mediante } \\
\text { técnicas } \\
\text { "Participativas". } \\
\text { Su participación como } \\
\text { sociedad: } \\
>\text { Disponibilidad al } \\
\text { cambio. }\end{array}$ \\
\hline
\end{tabular}

Fuente: Elaboración propia, en base a los talleres participativos.

Las personas del área forestal enfocan sus necesidades y expectativas al apoyo de programas de reforestación, siendo importante en ellos: inculcar mediante la participación ciudadana una cultura de seguimiento en proyectos elaborados por las instituciones, después del apoyo de algún programa de gobierno, que permita el logro 
Diagnóstico participativo para determinar problemas ambientales en comunidades rurales

de un progreso continuo y alcanzar así el desarrollo sustentable, sin generar un círculo vicioso de desorientación ambiental, en la comunidad. El resultado final fue el mapa participativo de los proyectos estratégicos que contribuyen al aprovechamiento sustentable de los recursos naturales, Figura 2.

\section{Principales conflictos ambientales}

En el Municipio de Álamos, son tres situaciones las que se identifican como las principales generadoras de conflicto: 1 . Escasez y disponibilidad de agua; 2. Delimitación de linderos (predios, ejidos, comunidades) y 3. Cultivo de enervantes.

Figura 2. Mapa participativo.

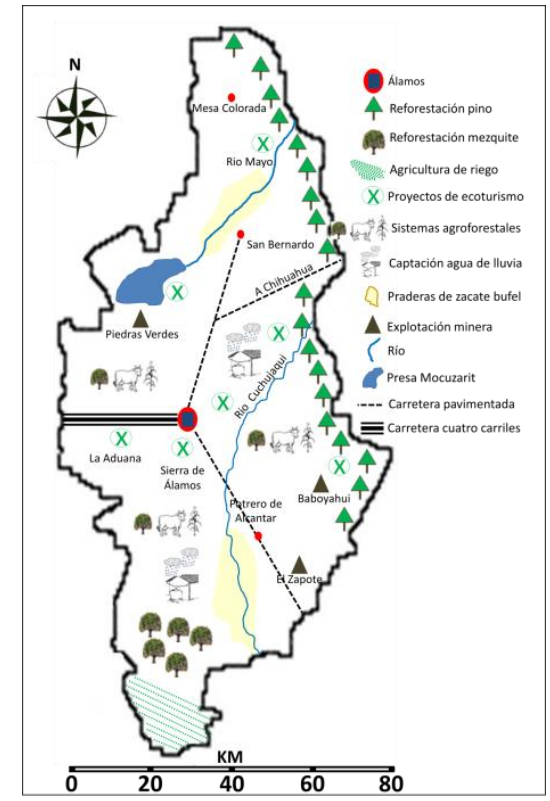

Fuente: Elaboración propia, en base a los talleres participativos.

1. Escasez y disponibilidad de agua. En este punto el conflicto está ubicado en diferentes escalas, iniciando con el de cuenca hidrográfica tanto la del Río Mayo como la del Arroyo Cuchujaqui, donde el consejo de cuenca es uno de los órganos que 
Adán Guillermo Ramírez-García y Mirna Camacho-Bercherlt

Telos Vol. 21, No. 1 (2019). 86-113

vigilan la administración del recurso entre los usuarios de la cuenca baja, quienes tienen prioridad sobre los que se ubican en la cuenca alta, siendo aquí justamente donde se origina el recurso en disputa; el siguiente nivel se da entre la ciudad de Álamos, que es a la vez la cabecera municipal y las comunidades de donde se abastece dicha ciudad; enseguida está la inconformidad que se genera entre las comunidades y la Comisión Nacional del Agua, institución encargada de emitir los permisos para la perforación de pozos y lograr el abasto de agua de uso doméstico y ganadero; finalmente está el conflicto entre algunas comunidades y la posibilidad de la explotación minera de cobre y la de plata.

a) Prioridades en las cuencas hidrológicas del río Mayo y el arroyo Cuchujaqui. Las cuencas hidrológicas del Río Mayo y el arroyo Cuchujaqui son relevantes por el aporte que hacen en los volúmenes de agua que se disponen en la zona de la planicie costera, donde se utiliza el agua captada en las presas del Mocúzari y la Josefa Ortiz de Domínguez en la agricultura de riego del Valle del Mayo, cuya superficie física de riego es de 95,000 hectáreas, en el área Fuerte-Mayo aun cuando de momento no rebasan las 3,000 hectáreas se tiene proyectada una superficie de 16,000 hectáreas; de ahí que el agua proporcionada por ambas cuencas se le dé prioridad de uso para la zona de riego y no en la captación que demandan constantemente los ganaderos de la cuenca alta, a través de la construcción de represas para abrevadero del ganado y en la perforación de pozos para explotar pequeñas áreas agrícola de riego.

b) El otro nivel de conflicto es el que se establece entre la cabecera municipal y las comunidades de donde se abastece de agua por medio del establecimiento de pozos de bombeo, ello conlleva a que las comunidades de donde proviene el agua se vean disminuidas en su servicio. Sin embargo, el problema no termina ahí, sino que una vez utilizada el agua, ésta no es tratada al salir como agua negra y se incorpora a la corriente subterránea del arroyo Cuchujaqui, provocando la contaminación de la corriente en mención; lo que también afecta aguas abajo a los usuarios que dependen del abasto de agua por bombeo para el uso doméstico y ganadero. 
Diagnóstico participativo para determinar problemas ambientales en comunidades rurales

c) Un tercer nivel lo constituye aquél que se genera entre las comunidades demandantes de agua en el municipio y la Comisión Nacional del Agua, al solicitar los permisos respectivos para la perforación de pozos; dichos permisos al parecer se ven sumamente restringidos argumentándose la existencia de áreas vedadas para la extracción de agua; no obstante lo anterior se tienen reportados 888 permisos de los cuales el primer lugar lo ocupan los de uso pecuario con el $39.64 \%$, le siguen los de uso múltiple con el $32.32 \%$, después están los de uso agrícola con el $15.99 \%$, en cuarto lugar están los de uso público urbano con el $7.43 \%$, después los de uso doméstico con el $3.60 \%$ y finalmente están los de servicios con el $0.90 \%$, al igual que los que generan electricidad con el $0.11 \%$.

d) El siguiente nivel de conflicto es el que se está iniciando entre algunas comunidades que se verán afectadas con la puesta en marcha de la actividad minera, lo que presupone requerirá de volúmenes de agua considerables y cuyos mantos freáticos aguas abajo se verán disminuidos.

En el caso de la empresa que explotará el mineral de cobre es probable que aun cuando el proceso de lavado no se lleve a cabo en la localidad, su impacto por efecto de la erosión hídrica y su depositación en la presa del Mocúzari se verá afectada la calidad de agua ahí almacenada, lo que constituirá un problema para su uso en las áreas de riego y en la producción pesquera de este cuerpo de agua.

Las disputas por el agua por lo menos en esta región han sido históricas, sin embargo ya no hay duda de que ha habido una baja en las precipitaciones que normalmente se tenían en la región, lo que pudiera tener relación con el deterioro de la flora y el suelo que se observa, así como con el cambio climático que se está registrando a nivel mundial, y localmente se manifiesta en la muerte de encinos y Guásima en sus respectivos hábitats locales; ello obliga a pensar que es urgente rediseñar nuevas estrategias que posibiliten el disponer de agua a mediano y largo plazo de manera sustentable, de ahí que las acciones deben planearse a nivel de toda la cuenca . 
Adán Guillermo Ramírez-García y Mirna Camacho-Bercherlt

Telos Vol. 21, No. 1 (2019). 86-113

2. Delimitación de linderos. La delimitación de linderos tanto a nivel municipal como entre comunidades y ejidos es otro tema que presenta cierta controversia, ello porque está implícito el usufructo de recursos naturales como: áreas con potencial agrícola, aguajes y la probable recaudación de impuestos.

a) Delimitación del área municipal. Aquí, la situación es que por un lado el INEGI reporta una superficie y una forma del municipio; sin embargo, ésta no corresponde con la que manejan los funcionarios del $\mathrm{H}$. Ayuntamiento, quienes aseguran que el municipio tiene una prolongación hacia el noroeste, lugar donde se encuentra asentada la etnia guarijío.

b) Delimitación de linderos entre ejidos y comunidades. El conflicto surge porque a veces no se encuentran los linderos respectivos, o en su momento no se definieron con exactitud éstos, aprovechando el conocimiento de las personas mayores a quienes les tocó el reparto agrario y fueron testigos presenciales. En el conflicto permea la calidad del terreno en disputa que puede ser para cultivo o bien para agostadero, así como la presencia de aguajes, lo que le da un valor relevante durante el período de sequía, es ahí donde abreva el ganado.

3. Cultivo de enervantes. El siguiente punto que llama la atención es el referido al cultivo de enervantes, aunque existe conciencia entre la sociedad y las autoridades municipales. Esto es producto de la alta marginación y pobreza que impera en el municipio, donde la falta de oportunidades de empleo y la reducida utilidad que presenta la ganadería, forestería y agricultura de subsistencia no alcanza para sufragar las necesidades mínimas familiares, lo que obliga a establecer dichos cultivos, a pesar del riesgo que conlleva. Otra salida, es la emigración a regiones localizadas en las áreas agrícolas de riego y en la frontera norte del estado para ocuparse como obreros en las maquiladoras ahí establecidas.

La prevalencia de condiciones de pobreza y marginación que caracterizan a esta región del sur del estado de Sonora, así como la falta de vías de comunicación y la 
Diagnóstico participativo para determinar problemas ambientales en comunidades rurales

dispersión de la población, ha provocado el incremento de actividades ilícitas desde vandalismo (asaltos, robos y secuestros) hasta la siembra de estupefacientes. Si bien esta última en cierto grado alivia las necesidades básicas de gente local, muchas veces provoca la pérdida irreparable de los elementos que conforman los ecosistemas y especies que caracterizan esta región.

\section{Conclusiones}

Sin duda, la evaluación rural participativa contribuyo a identificar los principales problemas ambientales en relación al aprovechamiento de los recursos naturales y los conflictos ambientales que se viven en el municipio de Álamos. Permitió la concientización y el intercambio de información entre los participantes en las diferentes actividades desarrolladas. En cuanto a los problemas ambientales identificados destacan: el sobrepastoreo debido a la explotación ganadera extensiva, erosión del suelo, tala clandestina, plagas y enfermedades en los bosques, contaminación del agua, así como la falta de empleos y el estancamiento económico de la región. Los conflictos ambientales identificados son tres: escasez y disponibilidad de agua, delimitación de linderos y el cultivo de enervantes. Sobre este último aspecto, en los talleres fue muy notorio que se considera un asunto que causa conflicto entre las comunidades del municipio, sin embargo, al tratar las alternativas o buscar cómo resolverlo, ya no había respuesta alguna, dando a entender que es un asunto que no se puede tratar con mayor profundidad, no así los otros problemas o conflictos, donde los participantes se mostraban entusiastas y planteaban propuestas que pudieran implementarse. Si bien es cierto identificaban las responsabilidades que debía tener cada uno de las entidades involucradas, quedaba claro que la participación de la comunidad será la base para el cambio.

En cuanto a los talleres participativos permitieron el contacto directo con los diferentes actores y puntos de vista sobre la problemática identificada en el municipio, así mismo son complemento de los que se pudo constatar en los recorridos de campo y 
Adán Guillermo Ramírez-García y Mirna Camacho-Bercherlt

Telos Vol. 21, No. 1 (2019). 86-113

las entrevistas con los informantes clave. El trabajar en diferentes talleres con objetivos particulares y participantes conocedores del tema a tratar, permitió el intercambio de experiencias y establecer un dialogo de saberes entre las instituciones, comunidades, académicos y sectores involucrados. En cuanto a las alternativas planteadas a la problemática identificada destaca el desarrollo de proyectos ecoturísticos, establecer sistemas de captación de agua de lluvia, promover la actividad ganadera desde una óptica del doble propósito.

Respecto a la metodología empleada es necesario apuntar que ésta lleva por principio incorporada la idea de un proceso integrado y lógico, obviamente la metodología no basta por sí misma para el logro de los objetivos, y en ocasiones es necesario el apoyo de otros recursos investigativos que la apoyen y complementen, pero esos apoyos deben ir claramente definidos en la estructura de la acción que estemos ejecutando, sobre todo en el papel que van a jugar en el estímulo de la participación y la reflexión, es decir deben ser metodológicamente coherentes, y no un grupo de técnicas desarticuladas y sin lógica, por lo que es necesario que en cualquier proceso participativo tomar en cuenta al menos los aspectos arriba mencionados.

De esta forma, se concluye que el diagnóstico debe ser considerado como un pilar en la propuesta de estrategias de desarrollo regional, resultado de la participación activa de los actores locales, además de brindar la oportunidad para elaborar proyectos de desarrollo viables para las comunidades involucradas. Se considera que en la realización de este trabajo se alcanzó satisfactoriamente el objetivo planteado, por lo que la sistematización de esta experiencia puede ser tomada como un referente para situaciones similares.

\section{Referencias Bibliográficas}

Bocco, Gerardo., Velázquez, Alejandro y Torres, Alejandro. (2000). Ciencia, comunidades indígenas y manejo de recursos naturales. Un caso de investigación participativa en México. Revista Interciencia, Volumen 25, No. 2. Venezuela (Pp. 64-70). 
Diagnóstico participativo para determinar problemas ambientales en comunidades rurales

Carabias, Julia y Provencio Enrique, Toledo Carlos. (1994). Manejo de recursos naturales y pobreza rural. Ciencias. Núm. 36, octubre-diciembre, (Pp. 85).

COESPO. (2010). Indicadores Demográficos y Socioeconómicos, Álamos, Sonora. Consejo Estatal de Población, Sonora.

Contreras, Armando., Lafraya, Susana., Lobillo, José., Soto, Pablo., Carles, Rodrigo. (1998). Diagnóstico Rural Participativo. (Primera edición) Editorial Grupo de Acción Comarcal La Serranía-Rincón de Ademuz. España.

Córdoba, Marcela, Gottret Verónica, López Tito, Montes Álvaro, Ortega Liudmila, Perry Santiago. (2004). Innovación participativa: experiencia con pequeños productores agrícolas en seis países en América Latina. (Primera edición) Editorial Red de Desarrollo Agropecuario. Chile.

Chambers, Robert. (1997). Whose Reality Counts? Putting the First Last. (First edition). Intermediate Technology Publications. England.

Chambers, Robert. (1983). Rural development: Putting the last first. (First edition). Prentice Hall Publications. England.

Estrella, Marisol. y Gaventa, John. (2001). ¿Quién da cuenta de la realidad? Monitoreo y Evaluación Participativa: Revisión bibliográfica. Material de Trabajo $N^{\circ}$ 70. Traducción Caudillo, R.D. y Alatorre, G. Institute of Development Studies. Brighton, Susex BN19. England.

FAO. (1996). The Internet and rural development: recommendations for strategy and activity. (Primera edición) Editorial FAO. Italia.

FAO. (2001). Conflictos y manejo de recursos naturales. (Primera edición) Editorial FAO. Italia.

García, María y Rodríguez, Adolfo. (2006). Capital Natural-Cultural y Participación Social en iniciativas de Ecoturismo Comunitario. Revista de Geografía Agrícola. Núm. 036. México (Pp. 29-41).

Geilfus, Frans. (1997). 80 herramientas para el Desarrollo Participativo. (Primera edición) Editorial IICA-GTZ. El Salvador.

Guzmán, Gloria., Alonso, Antonio., Pouliquen, Yann., y Sevilla, Eduardo. (1994). Las metodologías participativas de investigación: el aporte al desarrollo local endógeno. (Primera edición). Editorial Instituto de Sociología y Estudios Campesinos Universidad de Córdoba. España.

Hellin, Jon., y Badstue, Lone. (2006). Reduciendo la brecha entre la realidad de los investigadores y la de los agricultores. LEISA, Revista Agroecológica. Volumen 22. No. 3. Perú (Pp. 5- 8).

Herman, Rosa., Kandel, Susan., y Dimas, Leopoldo. (2003). Compensación por servicios ambientales $y$ comunidades rurales: Lecciones de las Américas y temas críticos para fortalecer estrategias comunitarias. (Primera edición). Editorial PRISMA. San Salvador.

INEGI (Instituto Nacional de Estadística, Geografía e Informática). (2011). Censo de Población y Vivienda, 2010 (Informe nacional y estatales), México. Disponible en: http://www.censo2010.org.mx 
Stephen, Kellert., Jai, Mehta., Syma, Ebbin., Laly, Lichtenfeld. (2000). Community Natural Resource Management: Promise, Rhetoric, and Reality. Society \& Natural Resources, 13:8, Estados Unidos (Pp. 705-715).

Lombera, Rocío. (2004). Planeación Estratégica Participativa para el Desarrollo Local. (Primera edición). Editorial Gobierno de Chiapas. México.

Miranda, Carlos y Matos, Aureliano. (2002). Desarrollo Rural Sostenible Enfoque Territorial: La Experiencia del IICA en Brasil. (Primera edición). Editorial IICA. Brasil.

Mormontoy, Henry., Castillo, Enrique., Muñoz, Mónica. (2006). Proceso de Planificación Participativa del uso de las tierras para el Ordenamiento Territorial. (Primera edición) Editorial Regional Office for Latin América and the Caribbean, FAO. Chile.

Ramírez, Adán Guillermo., Cervantes, Marta Concepción., Sánchez, Pastor., Monterroso, Alejandro Ismael., Gómez, Jesus David. (2012). El ordenamiento ecológico territorial participativo instrumento para el desarrollo rural sustentable: el caso del municipio de Álamos, Sonora, México. En Aguilar Robledo, Miguel; Delgado López, Enrique; Vázquez Solís, Valente Y Reyes Pérez, Oscar (Coordinadores), Ordenamiento Territorial y Participación Social: Problemas y Posibilidades. (Primera edición). Centro de Investigaciones en Geografía Ambiental. México.

Selman, Paul. (2004). Community participation in the planning and management of cultural landscapes. Journal of Environmental Planning and Management. Volumen47, 3. Reino Unido (Pp. 365-392).

Scheinfeld, Enrique. (1999). Proyectos de inversión y conflictos ambientales. (Primera edición). Editorial SEMARNAP-INE. México.

Toledo, Carlos y Bartra, Armando. (2000). Del círculo vicioso al círculo virtuoso. Cinco miradas al desarrollo sustentable de las regiones marginadas. (Primera edición). Editorial SEMARNAP-PIV. México. 